\title{
A Class of Maximal Inequalities for Conditional Demimartingales
}

\author{
Xia Lin, De Cheng Feng, YaLi Lu
}

\begin{abstract}
In this paper, based on conditional Fubini theorem and a maximal inequality for conditional demimartingales, we obtain a class of maximal inequalities for conditional demimartingales.

Keywords - demi(sub) martingales; conditional demi(sub) martingales; conditional Fubini theorem; maximal inequalities
\end{abstract}

\section{INTRODUCTION}

Newman and Wright [1] introduced the concepts of demimartingale and demi(sub)martingale. Definition 2 is due to Hadjikyriakou [2], since then, many scholars have studied it. For example, Christofides and Hadjikyriakou [3] established some maximal inequalities and asymptotic results for conditional demimartingales. Wang and Wang [4] obtained some maximal inequalities for conditional demi(sub)martingales and minimal inequalities for nonnegative conditional demimartingales. Feng and Gao [5] obtained conditional moment inequalities of conditional demisubmartingales. Feng, Wen and Yang [6] established a class of maximum inequalities of conditional demi(sub)martingales.Wang and $\mathrm{Hu}$ [7] established some maximal $\phi$-inequalities for nonnegative conditional demimartingales and some maximal inequalities for conditional demimartingales based on concave Young functions.

Inspired by reference [8], this paper first gave the definition of demi(sub)martingale and conditional demi(sub)martingale. On this basis, we further obtained a class of maximal inequalities for conditional demimartingales .

Notation and conventions. Throughout this paper, $\left\{X_{n}, n \geq 1\right\}$ or $\left\{S_{n}, n \geq 1\right\}$ denote a sequence of random variables defined on a fixed probability space $(\Omega, \mathrm{G}, \mathrm{P}), \quad E^{\mathrm{F}}(X)$ denote the conditional expectation of $X$, that is, $E^{F}(X)=E(X \mid F)$, where $F$ be a sub-s algebra of G. Let $X^{+}=\max (0, X), a \vee b=\max (a, b), I(A)$ denote the indicator function of the set $A, \mathrm{~S}_{0}=0, \log x=\log _{e} x=\ln x$, $\log ^{+} x=\ln (x \vee 1)$. Let $C$ denote the class of Orlicz functions, that is, unbounded, nondecreasing convex functions $f:[0, \infty) \rightarrow[0, \infty)$ with $f(0)=0$. Let $C^{\prime}=\left\{f \in C: f^{\prime}(0)=0, \frac{f^{\prime}(x)}{x}\right.$ is integrable on $(0, e)$ for some $e>0\}$.

Given $f \in C$ and $a \geq 0$, define

$$
\Phi_{a}(x)=\int_{a}^{x} \int_{a}^{s} \frac{f^{\prime}(r)}{r} d r d s, x>0 .
$$

Set $\Phi(x)=\Phi_{0}(x), x>0$.

\section{DEFINITION OF CONDITIONAL DEMIMARTINGALE}

Definition 1 Let $\left\{S_{n}, n \geq 1\right\}$ be a sequence of random variables defined on $L^{1}(\Omega, G, P)$. Assume that for $\mathrm{j}=1,2, \ldots$,

$$
E\left[\left(S_{j+1}-S_{j}\right) f\left(S_{1}, \ldots, S_{j}\right)\right] \geq 0
$$

for all coordinatewise nondecreasing functions $f$ such that the expectation is defined, Then $\left\{S_{n}, n \geq 1\right\}$ is called a demimartingale. If in addition the function $f$ is assumed to be nonnegative, the sequence $\left\{S_{n}, n \geq 1\right\}$ is called a demisubmartingale.

Definition 2 Let $\left\{S_{n}, n \geq 1\right\}$ be a sequence of random variables defined on $L^{1}(\Omega, G, P)$. Assume that for $1 \leq i<j<\infty$,

$$
E^{F}\left[\left(S_{j}-S_{i}\right) f S_{1}, S_{i} ., \geq\right)
$$

for all coordinatewise nondecreasing functions $f$ such that the expectation is defined, Then $\left\{S_{n}, n \geq 1\right\}$ is called a $F$-demimartingale. If in addition the function $f$ is assumed to be nonnegative, the sequence $\left\{S_{n}, n \geq 1\right\}$ is called a $F$-demisubmartingale.

It is easy to check that for all $i \geq 1$, (2) is equivalent to

$$
E^{F}\left[\left(S_{i+1}-S_{i}\right) f\left(S_{1}, \ldots, S_{i}\right)\right] \geq 0
$$

From the property of conditional expectations that $E[E(X \mid F)]=E(X)$ for any random variable $X$ with $E|X|<\infty$, it follows that $F-$ demi(sub)martingales 
defined on a proability space $(\Omega, G, P)$ are demi(sub)martingales on the proability space $(\Omega, G, P)$, but the converse is not true.

\section{II . MAIN RESULTS}

Lemma 1[6] Let $\left\{S_{n}, n \geq 1\right\}$ be an $F$ - demimartingale and $g$ be a nonnegative convex function satisfying $g(0)=0$ and $\operatorname{Eg}\left(S_{n}\right)<+\infty$ for every $n \geq 1$. Suppose that $\left\{c_{n}, n \geq 1\right\}$ is a positive nondecreasing sequence of $F$ - measurable random variables, then for any $F-$ measurable random variable $e>0$ a.s., $e P^{F}\left(\max _{1 \leq k \leq n} c_{k} g\left(S_{k}\right) \geq e\right) \leq c_{n} E^{F}\left(g\left(S_{n}\right) I\left(\max _{1 \leq k \leq n} c_{k} g\left(S_{k}\right) \geq e\right)\right)$ a.s.

Theorem 1 Let $\frac{1}{p}+\frac{1}{q}=1, p>1$. Assume that $\left\{S_{n}, n \geq 1\right\}$ is an $F$-demimartingale and $g$ is a nonnegative convex function satisfying $g(0)=0$ and $E\left(g\left(S_{k}\right)\right)^{p}<+\infty$ for every $k \geq 1$.

Suppose that $\left\{c_{n}, n \geq 1\right\}$ is a positive nondecreasing sequence of $F$ - measurable random variables and define $S_{n}{ }^{\max }=\max _{1 \leq k \leq n} c_{k} g\left(S_{k}\right)$. Then for $f \in C^{\prime}$,

$E^{F} f\left(S_{n}{ }^{\max }\right) \leq\left(E^{F}\left[c_{n} g\left(S_{n}\right)\right]^{p}\right)^{\frac{1}{p}}\left(E^{F}\left[\Phi^{\prime}\left(S_{n}{ }^{\max }\right)\right]^{q}\right)^{\frac{1}{q}}$ a.s. $(4)$

Proof. Since from lemma 1 , the conditional Fubini theorem and the conditional Hölder inequality, we can get that

$$
\begin{aligned}
E^{F} f\left(S_{n}{ }^{\max }\right) & =E^{F}\left(\int_{0}^{S_{n}{ }^{\max }} f^{\prime}(t) d t\right) \\
& =E^{F}\left(\int_{0}^{\infty} f^{\prime}(t) I\left(S_{n}{ }^{\max } \geq t\right) d t\right) \\
& =\int_{0}^{\infty} E^{F}\left(f^{\prime}(t) I\left(S_{n}{ }^{\max } \geq t\right)\right) d t \\
& =\int_{0}^{\infty} f^{\prime}(t) P^{F}\left(S_{n}{ }^{\max } \geq t\right) d t \\
& \leq \int_{0}^{\infty} f^{\prime}(t) \cdot{ }_{t} c_{n} E^{F}\left(g\left(S_{n}\right) I\left(S_{n}{ }^{\max } \geq t\right)\right) d t \\
& =\int_{0}^{\infty} \frac{f^{\prime}(t)}{t} E^{F}\left(c_{n} g\left(S_{n}\right) I\left(S_{n}{ }^{\max } \geq t\right)\right) d t \\
& =E^{F}\left(\int_{0}^{S_{n}{ }^{\max }} \frac{f^{\prime}(t)}{t} c_{n} g\left(S_{n}\right)\right) d t \\
& =E^{F}\left(c_{n} g\left(S_{n}\right) \Phi^{\prime}\left(S_{n}{ }^{\max }\right)\right) \\
& \leq\left(E^{F}\left[c_{n} g\left(S_{n}\right)\right]^{p}\right)^{\frac{1}{p}}\left(E^{F}\left[\Phi^{\prime}\left(S_{n}{ }^{\max }\right)\right]^{q}\right)^{\frac{1}{q}} \text { a.s. }
\end{aligned}
$$

Corollary 1 Assume that the condition for Theorem 1 is satisfied and $c_{k} \equiv 1$ for every $k \geq 1$, then

$$
E^{F} f\left(\max _{1 \leq k \leq n} g\left(S_{k}\right)\right) \leq\left(E^{F}\left[g\left(S_{n}\right)\right]^{p}\right)^{\frac{1}{p}}
$$

$\times\left(E^{F}\left[\Phi^{\prime}\left(\max _{1 \leq k \leq n} g\left(S_{k}\right)\right)\right]^{q}\right)^{\frac{1}{q}}$

Corollary 2 Assume that the condition for Theorem 1 is satisfied. Then for every $p>1$,

$$
\begin{gathered}
E^{F}\left(S_{n}{ }^{\max }\right)^{p} \leq\left(\frac{p}{p-1}\right)^{p} E^{F}\left[c_{n} g\left(S_{n}\right)\right]^{p} \\
E^{F}\left(S_{n}^{\max }\right) \leq \frac{e}{e-1}\left\{1+E^{F}\left(c_{n} g\left(S_{n}\right) \times \log ^{+}\left(c_{n} g\left(S_{n}\right)\right)\right)\right\} .
\end{gathered}
$$

Proof. By taking $f(x)=x^{p}, p>1$ in Theorem 1 , we get $\Phi^{\prime}(x)=\frac{p}{p-1} x^{p-1}$, Hence

$$
E^{F}\left(S_{n}^{\max }\right)^{p} \leq\left(\frac{p}{p-1}\right)^{p} E^{F}\left[c_{n} g\left(S_{n}\right)\right]^{p}
$$

Taking $f(x)=(x-1)^{+}$in Theorem 1 , it follows that $f^{\prime}(x)=1 \cdot I(x \geq 1)$. Therefore

$$
\begin{aligned}
E^{F}\left(S_{n}{ }^{\max }-1\right) & \leq E^{F}\left(S_{n}{ }^{\max }-1\right)^{+} \\
& \leq E^{F}\left(c_{n} g\left(S_{n}\right) \int_{0}^{S_{n}{ }^{\max }} \frac{I(r \geq 1)}{r} d r\right) \\
& =E^{F}\left(c_{n} g\left(S_{n}\right) \log ^{+} S_{n}^{\max }\right)
\end{aligned}
$$

By the inequality

$$
a \log ^{+} b \leq a \log ^{+} a+b e^{-1}, a>0, b>0,
$$

we have

$$
\begin{aligned}
E^{F}\left(S_{n}{ }^{\max }-1\right) & \leq E^{F}\left(c_{n} g\left(S_{n}\right) \log ^{+} c_{n} g\left(S_{n}\right)+e^{-1} S_{n}^{\max }\right) \\
& =E^{F}\left(c_{n} g\left(S_{n}\right) \log ^{+} c_{n} g\left(S_{n}\right)\right)+e^{-1} E^{F}\left(S_{n}{ }^{\max }\right)
\end{aligned}
$$

Hence

$$
E^{F}\left(S_{n}^{\max }\right) \leq \frac{e}{e-1}\left\{1+E^{F}\left(c_{n} g\left(S_{n}\right) \times \log ^{+}\left(c_{n} g\left(S_{n}\right)\right)\right)\right\} .
$$

Let $c_{k} \equiv 1, k \geq 1$ in inequalities (6) and (7), we have

$$
\begin{aligned}
& E^{F}\left[\max _{1 \leq k \leq n} g\left(S_{k}\right)\right]^{p} \leq\left(\frac{p}{p-1}\right)^{p} E^{F}\left[g\left(S_{n}\right)\right]^{p} \text { a.s. } \\
& E^{F}\left[\max _{1 \leq k \leq n} g\left(S_{k}\right)\right] \leq \frac{e}{e-1}\left\{1+E^{F}\left[g\left(S_{n}\right) \times \log ^{+}\left(g\left(S_{n}\right)\right)\right]\right\} \quad \text { a.s. (9) }
\end{aligned}
$$

Remark 1 Letting $g(x)=|x|$ in inequality (7), we have

$E^{F}\left[\max _{1 \leq k \leq n} c_{k}\left|S_{k}\right|\right] \leq \frac{e}{e-1}\left\{1+E^{F}\left[c_{n}\left|S_{n}\right| \times \log ^{+}\left(c_{n}\left|S_{n}\right|\right)\right]\right\}$ 
Taking $g(x)=|x|$ in inequalities (8) and (9), we can get the following corollary.

Corollary 3 Let $\left\{S_{n}, n \geq 1\right\}$ be an $F$-demimartingale and $p>1$. Suppose that $E\left|S_{k}\right|^{p}<\infty$ for each $k \geq 1$, then

$$
\begin{aligned}
& E^{F}\left(\max _{1 \leq k \leq n}\left|S_{k}\right|\right)^{p} \leq\left(\frac{p}{p-1}\right)^{p} E^{F}\left|S_{n}\right|^{p} \quad \text { a.s. } \\
& E^{F}\left(\max _{1 \leq k \leq n}\left|S_{k}\right|\right) \leq \frac{e}{e-1}\left\{\begin{array}{l}
1+E^{F}\left(\left|S_{n}\right|\right. \\
\left.\times \log ^{+}\left|S_{n}\right|\right)
\end{array}\right\} \quad \text { a.s. }
\end{aligned}
$$

Theorem 2 Let $\left\{S_{n}, n \geq 1\right\}$ be an $F$-demimartingale and $g$ be an nonnegative function satisfying $g(0)=0$.

Assume that $\left\{c_{n}, n \geq 1\right\}$ is a positive nondecreasing sequence of $F-$ measurable random variable. Then for all $n \geq 1, t>0$ and $0<l<1$,

$$
\begin{aligned}
P^{F}\left(\max _{1 \leq k \leq n} c_{k} g\left(S_{k}\right) \geq t\right) & \leq \frac{l}{(1-l) t} \int_{t}^{\infty} P^{F}\left(c_{n} g\left(S_{n}\right) \geq l s\right) d s \\
& =\frac{l}{(1-l) t} E^{F}\left(\frac{c_{n} g\left(S_{n}\right)}{l}-t\right)^{+}
\end{aligned}
$$

Furthermore, for $f \in C^{\prime}, n \geq 1, a>0, b>0$ and $0<l<1$,

$$
\begin{aligned}
E^{F}\left(f\left(\max _{1 \leq k \leq n} c_{k} g\left(S_{k}\right)\right)\right) & =f(b)+\frac{l}{1-l} E^{F}\left\{\begin{array}{l}
\Phi_{b}\left(\frac{c_{n} g\left(S_{n}\right)}{l}\right) \\
I\left(c_{n} g\left(S_{n}\right) \geq l b\right)
\end{array}\right\} \\
& \left.=f(b)+\frac{l}{1-l} E^{F}\left\{\begin{array}{l}
\left(\Phi_{a}\left(\frac{c_{n} g\left(S_{n}\right)}{l}\right)-\Phi_{a}(b)\right) \\
-\Phi_{a}^{\prime}(b)\left(\frac{c_{n} g\left(S_{n}\right)}{l}-b\right) \\
l\left(c_{n} g\left(S_{n}\right) \geq l b\right)
\end{array}\right)\right\}
\end{aligned}
$$

Proof. Since from lemma 1 , we have

$$
\begin{aligned}
P^{F}\left(\max _{1 \leq k \leq n} c_{k} g\left(S_{k}\right) \geq t\right) & \leq \frac{1}{t} c_{n} E^{F}\left(g\left(S_{n}\right) I\left(\max _{1 \leq k \leq n} c_{k} g\left(S_{k}\right) \geq t\right)\right) \\
& =\frac{1}{t} E^{F}\left(c_{n} g\left(S_{n}\right) I\left(\max _{1 \leq k \leq n} c_{k} g\left(S_{k}\right) \geq t\right)\right) \\
& =\frac{1}{t} \int_{0}^{\infty} P^{F}\left(\max _{1 \leq k \leq n} c_{k} g\left(S_{k}\right) \geq t, c_{n} g\left(S_{n}\right) \geq s\right) d s \\
& \leq \frac{1}{t} \int_{0}^{l t} P^{F}\left(\max _{1 \leq k \leq n} c_{k} g\left(S_{k}\right) \geq t\right) d s \\
& +\frac{1}{t} \int_{l t}^{\infty} P^{F}\left(c_{n} g\left(S_{n}\right) \geq s\right) d s \\
& =l P^{F}\left(\max _{1 \leq k \leq n} c_{k} g\left(S_{k}\right) \geq t\right) \\
& +\frac{l}{t} \int_{t}^{\infty} P^{F}\left(c_{n} g\left(S_{n}\right) \geq l s\right) d s
\end{aligned}
$$

then

$(1-l) P^{F}\left(\max _{1 \leq k \leq n} c_{k} g\left(S_{k}\right) \geq t\right) \leq \frac{l}{t} \int_{t}^{\infty} P^{F}\left(c_{n} g\left(S_{n}\right) \geq l s\right) d s$ a.s.
So we have

$$
P^{F}\left(\max _{1 \leq k \leq n} c_{k} g\left(S_{k}\right) \geq t\right) \leq \frac{l}{(1-l) t} \int_{t}^{\infty} P^{F}\left(c_{n} g\left(S_{n}\right) \geq l s\right) d s
$$

It can be checked that

$$
\int_{t}^{\infty} I\left(\frac{c_{n} g\left(S_{n}\right)}{l} \geq s\right) d s=\left(\frac{c_{n} g\left(S_{n}\right)}{l}-t\right)^{+}, t \geq 0
$$

Hence

$$
\int_{t}^{\infty} P^{F}\left(c_{n} g\left(S_{n}\right) \geq l s\right) d s=E^{F}\left(\frac{c_{n} g\left(S_{n}\right)}{l}-t\right)^{+} \text {a.s. }
$$

Furthermore, the first inequality in theorem 2 is proved.

By the inequality that we got before, so we have

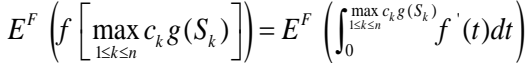

$$
\begin{aligned}
& =E^{F}\left(\int_{0}^{\infty} f^{\prime}(t) I\left(\max _{1 \leq k \leq n} c_{k} g\left(S_{k}\right) \geq t\right) d t\right) \\
& =\int_{0}^{\infty} E^{F}\left[f^{\prime}(t) I\left(\max _{1 \leq k \leq n} c_{k} g\left(S_{k}\right) \geq t\right)\right] d t \\
& =\int_{0}^{\infty} f^{\prime}(t) P^{F}\left(\max _{1 \leq k \leq n} c_{k} g\left(S_{k}\right) \geq t\right) d t \\
& =\int_{0}^{b} f^{\prime}(t) P^{F}\left(\max _{1 \leq k \leq n} c_{k} g\left(S_{k}\right) \geq t\right) d t \\
& +\int_{b}^{\infty} f^{\prime}(t) P^{F}\left(\max _{1<k \leq n} c_{k} g\left(S_{k}\right) \geq t\right) d t \\
& \leq f(b)+\int_{b}^{\infty} f^{\prime}(t) P^{F}\left(\max _{1 \leq k \leq n} c_{k} g\left(S_{k}\right) \geq t\right) d t \\
& \leq f(b)+\int_{b}^{\infty} f^{\prime}(t)\left[\frac{l}{(1-l) t} \int_{t}^{\infty} P^{F}\left(c_{n} g\left(S_{n}\right) \geq l s\right) d s\right] d t \\
& =f(b)+\frac{l}{1-l} \int_{b}^{\infty} \frac{f^{\prime}(t)}{t}\left[\int_{t}^{\infty} P^{F}\left(c_{n} g\left(S_{n}\right) \geq l s\right) d s\right] d t \\
& =f(b)+\frac{l}{1-l} \int_{b}^{\infty} \frac{f^{\prime}(t)}{t} E^{F}\left(\frac{c_{n} g\left(S_{n}\right)}{l}-t\right)^{+} d t \\
& =f(b)+\frac{l}{1-l} E^{F}\left[\int_{b}^{\infty} \frac{f^{\prime}(t)}{t}\left(\frac{c_{n} g\left(S_{n}\right)}{l}-t\right)^{+} d t\right] \\
& =f(b)+\frac{l}{1-l} E^{F}\left(\int_{b}^{\infty} \frac{f^{\prime}(t)}{t}\left[\int_{t}^{\infty} I\left(\frac{c_{n} g\left(S_{n}\right)}{l} \geq s\right) d s\right] d t\right) \\
& =f(b)+\frac{l}{1-l} E^{F}\left(\int_{b}^{\frac{c_{n} g\left(S_{n}\right)}{l}}\left[\int_{b}^{s} \frac{f^{\prime}(t)}{t} d t\right] d s \cdot I\left(c_{n} g\left(S_{n}\right) \geq l b\right)\right) \\
& =f(b)+\frac{l}{1-l} E^{F}\left(\Phi_{b}\left[\frac{c_{n} g\left(S_{n}\right)}{l}\right] I\left(c_{n} g\left(S_{n}\right) \geq l b\right)\right)
\end{aligned}
$$

Since

$$
\begin{aligned}
& \Phi_{a}\left(\frac{c_{n} g\left(S_{n}\right)}{l}\right)-\Phi_{a}(b)-\Phi_{b}\left(\frac{c_{n} g\left(S_{n}\right)}{l}\right)=\int_{a}^{\frac{c_{n} g\left(S_{n}\right)}{l}} \int_{a}^{s} \frac{f^{\prime}(r)}{r} d r d s \\
& -\int_{a}^{b} \int_{a}^{s} \frac{f^{\prime}(r)}{r} d r d s-\int_{b}^{c_{n} g\left(S_{n}\right)} l=\int_{b}^{s} \frac{f^{\prime}(r)}{r} d r d s \\
& =\int_{b}^{\frac{c_{n} g\left(S_{n}\right)}{l}} \int_{a}^{s} \frac{f^{\prime}(r)}{r} d r d s-\int_{b}^{\frac{c_{n} g\left(S_{n}\right)}{l}} \int_{b}^{s} \frac{f^{\prime}(r)}{r} d r d s \\
& =\int_{b}^{\frac{c_{n} g\left(S_{n}\right)}{l}} \int_{a}^{b} \frac{f^{\prime}(r)}{r} d r d s \\
& =\int_{a}^{b} \frac{f^{\prime}(r)}{r} d r\left(\frac{c_{n} g\left(S_{n}\right)}{l}-b\right) \\
& =\Phi_{a}^{\prime}(b)\left(\frac{c_{n} g\left(S_{n}\right)}{l}-b\right)
\end{aligned}
$$

Hence, the second inequality in theorem 2 is proved In conclusion, theorem 2 is proved. 
Remark 2 Taking $c_{k} \equiv 1$ for each $k \geq 1$ in Theorem

2 , then

$$
\begin{aligned}
P^{F}\left(\max _{1 \leq k \leq n} g\left(S_{k}\right) \geq t\right) & \leq \frac{l}{(1-l) t} \int_{t}^{\infty} P^{F}\left(g\left(S_{n}\right) \geq l s\right) d s \\
& =\frac{l}{(1-l) t} E^{F}\left(\frac{g\left(S_{n}\right)}{l}-t\right)^{+}
\end{aligned}
$$

Furthermore, for $f \in C^{\prime}, n \geq 1, a>0, b>0$ and

$0<l<1$

$$
\begin{aligned}
E^{F}\left(f\left(\max _{1 \leq k \leq n} g\left(S_{k}\right)\right)\right) & =f(b)+\frac{l}{1-l} E^{F}\left(\Phi_{b}\left(\frac{g\left(S_{n}\right)}{l}\right) I\left(g\left(S_{n}\right) \geq l b\right)\right) \\
& =f(b)+\frac{l}{1-l} E^{F}\left\{\left(\begin{array}{l}
\left.\left(\Phi_{a}\left(\frac{g\left(S_{n}\right)}{l}\right)-\Phi_{a}(b)\right)\right) \text { a.s. } \\
\left.\left.-\Phi_{a}^{\prime}(b)\left(\frac{g\left(S_{n}\right)}{l}-b\right)\right)\right\} \\
I\left(g\left(S_{n}\right) \geq l b\right)
\end{array}\right)\right.
\end{aligned}
$$

\section{ACKNOWLEDGEMENT}

This work is supported by National Natural Science Foundation of China and Innovation ability improvement project of colleges and universities in Gansu Province and Graduate research self-help project of Northwest Normal University (Grant No. 11861057 and Grant No. 11761064 and Grant No.2019A-003 and Grant No.2020KYZZ001113 ).

\section{REFERENCES}

[1] NEWMAN,C M.WRIGHT,A L.Associated random variables and martingale inequalities[J].Z Wahrsch Verw Geb,1982,59(3):36 1-371.

[2] HADJIKYRIAKOU,M. Probability and moment inequalities for demimartingales and associated random variables[D]. Nicosia: Department of Mathematics and Statistics, University of Cypru s, 2010 .

[3] CHRISTOFIDES,T.C. and Hadjikyriakou,M. Conditional demima rtingales and related results[J]. Journal of Mathematical Analy is and Applications,2013,398(1):380-391.

[4] WANG X.H, WANG X.J. Some inequalities for conditional dem imartingales and conditional N-demimartingales[J]. Statistics an d Probability Letters,2013,83(3):700-709.

[5] FENG Decheng, GAO Yufeng. Conditional moment ineualities of conditional demisubmartingales[J]. Journal of Lanzhou Univer sity of Technology,2017,43(1):154-156.

[6] FENG Decheng, WEN Huimin, YANG Yanan. A class of maxi mum inequalities of conditional demi(sub)martingales[J]. Journ al of Lanzhou University of Technology,2020,46(1):158-161.

[7] WANG X.H, HU S.H. On the maximal inequalities for conditio nal demimartingales[J]. Journal of Mathematical Inequalities, 2 014,8(3):545-558.

[8] X.B. GONG. Maximal $\phi$-inequalities for demimartingales[J]. Ine
qual.Appl,2011,59.

Xia Lin, School of Mathematics and Statistics, Northwest Normal University,Lanzhou,China,Mobile 86-18298360284.

DeCheng Feng, School of Mathematics and Statistics, Northwest Normal University, Lanzhou, China.

YaLi Lu, School of Mathematics and Statistics, Northwest Normal University, Lanzhou, China. 